\title{
Patterns and Surgical Outcome of Pediatric Intestinal Obstruction in Pakistan
}

\author{
Ali Raza Chaudhry, Muhammad Umar Nisar ${ }^{1}$, Anwar Khan ${ }^{2}$, Nadeem Akhtar $^{1}$, Samer Sikander ${ }^{3}$, \\ Nisar Ahmed Khan ${ }^{4}$
}

\section{ABSTRACT}

Background: Intestinal obstruction in children is a very common cause of admission in hospitals with usually a high mortality and morbidity rate. There is a geographical variability in patterns of intestinal obstruction in pediatric population around the globe.

Objectives: To evaluate patterns of presentation and surgical outcome of pediatric intestinal obstruction in Pakistan.

Material and Methods: A prospective observational study with non-probability sampling technique was done in the department Pediatric Surgery (East Surgical Unit), The Children's Hospital, Pakistan Institute of Medical Sciences (PIMS), Islamabad, Pakistan, from January to December 2017. Operated cases of intestinal obstruction from 1 day to 12 years were included. Age, patterns of presentation, sign and symptoms, surgical intervention, complications, outcome and correlation between variables were studied.

Results: Total cases were 316 (231 males, 85 females). Congenital Causes of intestinal obstruction in descending order were Anorectal Malformations(75), Jejuno-Ileal Atresia(28), Hirschsprung Disease(27), Meckel's Diverticulum(17), Duodenal Atresia(16), Malrotation(12), Meconium lleus(11), Midgut Volvulus(8), Mesenteric cyst(1) and Rectal Atresia(1). Acquired causes were Intussusception(40), Perforated Appendix(20), Infantile Hypertrophic Pyloric Stenosis(14), Band Obstruction(12), Blunt Abdominal Trauma(10), Obstructed Inguinal hernia (9), Worm infestation(9), Enteric Perforation(3), Necrotizing Enterocolitis(3). The overall mortality rate was $3.5 \%$ and postoperative complications rate was $40.8 \%$. Statistically significant relationship was found between Age and post operative complications $(P=0.048)$, Age and outcome $(P=0.002)$ and between post operative complications and outcome $(p<0.001)$.

Conclusions: Causes of intestinal obstruction vary according to the age and geographical area. Accurate and timely diagnosis of the cause of intestinal obstruction along with prevention and treatment of sepsis can reduce morbidity and mortality.

Key words: Intestinal obstruction; Children; Congenital; Acquired Causes; Neonate; Pakistan

This article may be cited as: Chaudhry AR, Nisar MU, Khan A, Akhtar N, Sikander S, Khan NA.

Patterns and Surgical Outcome of Pediatric Intestinal Obstruction in Pakistan. J Saidu Med Coll Swat 2021;11(1):39-44

\section{INTRODUCTION}

Intestinal obstruction (IO) is one of the most common surgical problems encountered in pediatric emergency department. Over the past few decades, due to better understanding of pathophysiology, advanced diagnostics, better antibiotic availability, safe pediatric anesthesia and improved pre and post operative care, the diagnosis and management in pediatric intestinal obstruction has changed a lot. ${ }^{1}$

IO is defined as impedance of intestinal contents to flow that can be either due to congenital or acquired causes. There are various causes of $I O$ in pediatric age group. The difference in pathophysiology of IO between adult and pediatric population is that congenital causes play a major role in case of pediatric population. Some common causes of pediatric 10 described in literature include Anorectal Malformations (ARM),

\footnotetext{
1. Resident Paediatric Surgery, The Children's Hospital, Pakistan Institute of Medical Sciences, Islamabad, Pakistan.

2.Consultant Pediatrics Surgeon, Saidu Group of Teaching hospital, Swat, Pakistan

3. Resident Paediatric Medicine, PAEC General Hospital Islamabad

4. Associate Professor, Community Medicin, Mohiuddin Islamic Medical College, Mirpur, Azad Jammu \& Kashmir

Correspondence: Ali Raza Chaudhry

Resident Pediatric Surgery Children Hospital, Pakistan Institute of Medical Sciences, Islamabad, Pakistan

Phone: +923235506688Email: alirchaudhry@gmail.com
}

Received: September 18, 2019 Accepted: November 23, 2020
Hirschsprung disease, Malrotation, volvulus, intestinal atresia, intussusception and infantile hypertrophic pyloric Stenosis (IHPS).,

Age remains an important factor in diagnosis of various causes of IO. For example, IO secondary to congenital causes such as ARM, Meconium ileus, intestinal atresia, long segment Hirschsprung disease and malrotation with or without volvulus present mostly in early neonatal age soon after birth, whereas, most acquired causes such as intussusception present at later age. ${ }^{3}$ Inability to pass meconium within 24 hours in a full term neonate should raise the suspicion of intestinal obstruction. Other signs and symptoms of IO include, pain abdomen, vomiting, abdominal distension, failure to pass meconium/stool. Early detection of cause of $\mathrm{IO}$ in pediatric population is of paramount importance in order to avoid undue morbidity and mortality. Delay in diagnosis can lead to fluid and electrolyte imbalance, perforation, peritonitis, aspiration pneumonitis, sepsis and death. ${ }^{1,4}$

There is a great geographical variability in patterns of presentation of $\mathrm{IO}$ in pediatric population around the globe. ${ }^{1,5}$ Unfortunately, there is a paucity of data regarding patterns of presentation and surgical outcome of pediatric intestinal obstruction in Pakistan. The aim of this 
study is to evaluate characteristics, patterns of presentation and outcome of $\mathrm{IO}$ in pediatric population in a developing country. By understanding these patterns and challenges unique to this geographical region, strategies can be devised to reduce intestinal obstruction related pediatric morbidity and mortality in Pakistan.

\section{MATERIALAND METHODS}

This is a prospective observational study with nonprobability sampling technique. The study was conducted in the Department of Pediatric Surgery (East Surgical Unit), The Children's Hospital, Pakistan Institute of Medical Sciences (PIMS), Islamabad, Pakistan. A total number of 316 operated cases of intestinal obstruction from 1 day of age to 12 years were included. Patients above 12 years of age, patients of $I O$ who were managed conservatively and patients with dynamic obstruction were excluded. The study period was from $1^{\text {st }}$ January 2017 to $31^{\text {st }}$ December 2017. All the patients were admitted through emergency.

Sample size was calculated using WHO calculator in which the population proportion was $25 \%$ \& precision was taken as $5 \%$, with confidence level of $95 \%{ }^{2}$

Parameters studied include name, age and gender, signs and symptoms, type of operation, post-operative complications and outcome as survival or mortality.

Depending on availability of resources standard management was given to all the patients. Resuscitation was done in those who were sick at presentation. Baseline investigations of Complete Blood Count, liver function tests, PT, INR, Urea, Creatinine and serum electrolytes were done in all the cases. Radiological investigations included Plain $x$-ray abdomen erect and supine and Ultrasonography (USG) of abdomen and pelvis. Computed tomography (CT) scan was done in cases where diagnosis was in doubt. Water soluble contrast study was done where Hirschsprung disease was in differential diagnoses. Cases were operated by professors and senior postgraduate residents (R5). Standard post operative management was done and patients were discharged home when they were stable vitally, tolerating orally and passing stool. All the patients were followed up for 4 weeks to look for any post operative complication.
Data analysis was done using SPSS version 21. Descriptive statistics like mean and standard deviation for quantitative data and frequency and percentages for qualitative data were calculated. Chi-square test was applied to associate different parameters with surgical outcome in terms of discharge and died. P-value $<0.05$ was considered significant.

A written informed consent was taken from parents/guardians and the study was approved by institutional ethical review board.

\section{RESULTS}

The number of all operated cases in our study was 316 . In our study $231(73.1 \%)$ patients were male and $85(26.9 \%)$ were female. Age of patients in this series ranged from 1 day of life to 12 years. Patients with age range from 0-28 days were 175 (55.4\%), 29 days-12 months were 67 (21.2\%), 13 months to 12 years were $74(23.4 \%)$.

All the patients were explored via open method. No laparoscopic or minimally invasive surgical techniques were used. In our study congenital causes of intestinal obstruction were more common than acquired causes Table 1 . Out of 316 patients, 179 (56.6\%) patients had congenital causes and 137 (43.4\%) patients had acquired causes. Anorectal Malformations $75(23.7 \%$ ) was the most common cause of congenital obstruction in our series. Among acquired causes, Intussusception 40(12.7\%) was the leading cause. Details of causes of intestinal obstruction are shown in Table 2.

Pain abdomen was the most common symptom in our study. $230(72.7 \%)$ patients presenting with intestinal obstruction had pain abdomen. The second most common symptom was abdominal distension. 213(67.4\%) patients had abdominal distension as a presenting symptom. Details of other presenting symptoms are shown in Fig 1.

Among 14 patients with pyloric stenosis 12 had Ramstedt Pyloromyotomy and 2 had to undergo gastrojejunostomy because of mucosal breach. Among 15 patients of ileal atresia 11 had enteroenterostomy and 4 underwent Bishop Koop. All 13 patients with jejunal atresia were treated by entero-enterostomy. Ladd's procedure was done in all 12 cases of malrotation. In 17 cases of Meckel's diverticulum, resection of Meckel's diverticulum was done followed by an end to end 
Table 1. Distribution of cases according to Gender

\begin{tabular}{lrr}
\hline Aetiology & Sex & \\
\hline & Female & Male \\
Congenital & 44 & 135 \\
Acquired & 41 & 96 \\
Total (316) & 85 & 231 \\
\hline
\end{tabular}

Table 2. Distribution of cases according to Etiological Incidence

\begin{tabular}{|c|c|c|c|c|c|}
\hline Congenital Causes & Frequency & $\begin{array}{c}\text { Percent } \\
(\%)\end{array}$ & Acquired Causes & Frequency & $\begin{array}{c}\text { Percent } \\
(\%)\end{array}$ \\
\hline Anorectal Malformation & 75 & 23.7 & Intussusception & 40 & 12.6 \\
\hline Jejuno-Ileal Atresia & 28 & 4.7 & Perforated Appendix & 20 & 6.3 \\
\hline Hirschsprung Disease & 27 & 8.5 & $\begin{array}{l}\text { Hypertrophic Pyloric } \\
\text { Stenosis }\end{array}$ & 14 & 4.4 \\
\hline Duodenal Atresia & 16 & 5 & Band Obstruction & 12 & 3.7 \\
\hline Meckels Diverticulum & 17 & 5.3 & Blunt abdominal trauma & 10 & 3.1 \\
\hline Malrotation & 12 & 3.7 & Obstructed inguinal hernia & 9 & 2.8 \\
\hline Meconium lleus & 11 & 3.4 & Worm Infestation & 9 & 2.8 \\
\hline Midgut Volvulus & 8 & 2.5 & Enteric Perforation & 3 & 0.9 \\
\hline Mesenteric Cyst & 1 & 0.3 & Necrotizing enterocolitis & 3 & 0.9 \\
\hline Rectal Atresia & 1 & 0.3 & & & \\
\hline
\end{tabular}

anastomosis. Among 11 patients of meconium ileus, 8 underwent Bishop Koop and 3 had double barrel stoma formation. A single case of mesenteric cyst induced obstruction was treated by resection followed by an end to end anastomosis. There were 8 cases of Acute Midgut Volvulus secondary to malrotation. 4 of them had resection and anastomosis, 2 had stoma formation and remaining 2 underwent Ladd's procedure. All three cases of necrotizing enterocolitis had stoma formation. There were 9 cases of obstructed inguinal hernia, 8 had emergency herniotomy and one underwent resection and anastomosis. 19 out 20 perforated appendix were treated with appendectomy and one with stoma formation. A single case of rectal atresia in our cohort underwent stoma formation. Among 9 patients with worm infestation, 5 had to undergo enterotomy for removal of worms and 4 underwent resection secondary to gangrenous bowel.

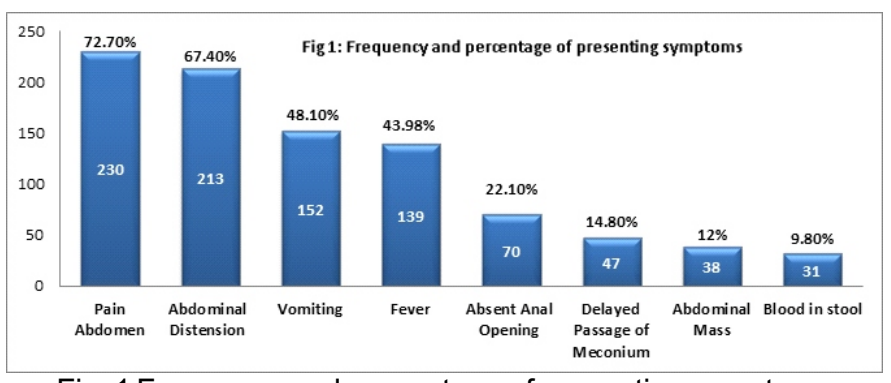

Fig. 1 Frequency and percentage of presenting symptoms

There were 11(3.5\%) deaths and 129 (40.8\%) patients had postoperative complications. The most common postoperative complication was Sepsis in $61(19.3 \%)$ patients which was managed with IV antibiotics according to culture and sensitivity, Surgical site infection in 24 (7.69\%), patients, wound dehiscence in $12(3.80 \%)$ patients and prolapsed stoma in $5(1.6 \%)$ patients. Fig: 2 
When outcome was analyzed in relation to post operative complications, statistically significant correlation was found ( $p<0.001$ ). Eight out of eleven patients with post operative complications died. Sepsis (8 patients) was the main cause of mortality in patients with post operative complications. Statistically significant relationship was found when age was compared to post operative complications $(p=0.001)$. Neonatal age group had highest rate of post operative complications. Sixty three out of 175 neonates have post operative complications and again sepsis (46 patients) was the major complication in neonatal age group. Statistically significant relationship was found when age is compared to outcome $(p=0.048)$. Ten out of eleven deaths occurred in neonatal age.

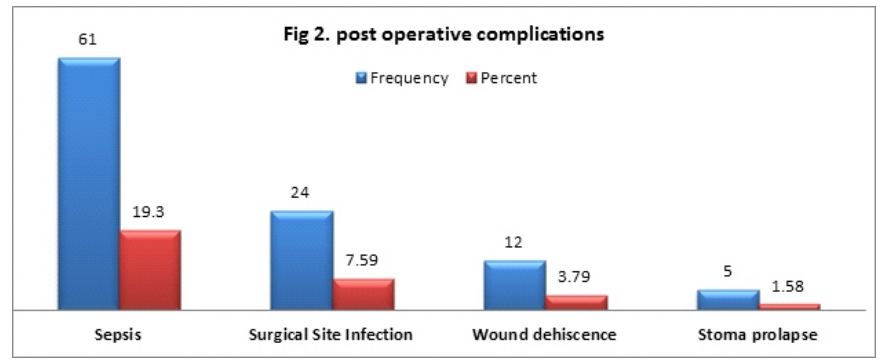

\section{DISCUSSION}

Intestinal obstruction in neonatal age may be due to various causes and these causes should be in the mind of every pediatrician or a physician attending these newborns. Anorectal malformation is the commonest congenital cause of intestinal obstruction in neonates. Other common surgical causes in descending order include duodenal atresia, Hirschsprung's disease, meconium ileus and meconium plug syndrome. ${ }^{6}$ In our study anorectal malformation was the most common congenital cause of intestinal obstruction $(21.4 \%)$ followed by Jejuno-ileal atresia, Hirschsprung disease and malrotation respectively. In a study from Jammu \& Kashmir, ARM was found to be the most common neonatal IO $(25 \%)$ followed by Jejuno ileal atresia $(20 \%)$ and duodenal atresia. ${ }^{4}$ Another study from India showed ARM being most common cause of neonatal IO. ${ }^{1}$ Similar pattern of presentation was shown in a study from Bangladesh with ARM being the most common cause followed by Hirschsprung disease. ${ }^{3}$ These results show similarity in patterns of presentation of $\mathrm{IO}$ in the same geographical area.
Neonates and infants have limited physiological reserves and delay in diagnosis may result in undue morbidity and mortality. In developing countries, these cases are usually attended by a general physician or a pediatrician and if these cases are not picked up and referred to the pediatric surgeon early, may result in an increase in morbidity and mortality.

Intestinal obstruction in neonates is almost always congenital and diagnosing the causes is a major challenge for neonatologist and pediatric surgeons all over the world. They constitute the major portion of surgical admissions in children hospitals as in our study 175 out of 316 patients $(55.4 \%)$ fall into this category. Failure to pass meconium, abdominal distension, bilious vomiting were the common presenting features. Episodes of bilious vomiting should always be considered significant and raise the suspicion of a surgical cause until and unless proven otherwise. If there are no other suspected medical causes such as hypothyroidism or sepsis, failure to pass meconium in a full term healthy neonate should raise the suspicion of intestinal obstruction. Abdominal distension may be a prominent feature of distal bowel obstruction. In proximal obstruction, there may only be epigastric fullness. Abdominal distension may also be a feature of complications of intestinal obstruction such as gut perforation in which a neonate may present with the hugely distended abdomen. Neonate with necrotizing enterocolitis may also present with hugely distended and tense abdomen. Bleeding per rectum is a sign of bowel ischemia and requires early intervention. ${ }^{7}$ Older children with intestinal obstruction also present with similar features of pain abdomen, constipation, vomiting and abdominal distension.

Older children may present with intestinal obstruction secondary to intussusception, perforated appendix, enteric perforation, blunt abdominal trauma, Meckel's diverticulum, obstructed hernia, intestinal ascariasis and other rare causes.

Intussusception is the common acquired cause of intestinal obstruction in infants and young children resulting in significant morbidity. It occurs more commonly in male than females. Cause of intussusception is idiopathic in $90 \%$ of the cases. Only $10 \%$ have definite pathological lead point 
such as Meckel's diverticulum, lymphoid hyperplasia, inflamed appendix, mass, abdominal cysts, resulting in intussusception. It shows seasonal variability with peak incidence coinciding with the incidence of respiratory tract infections and diarrhea. ${ }^{8,9}$ In our study intussusception was the most common acquired cause of intestinal obstruction (12.6\%). In a study conducted by Ooko et al, intussusception was the most common cause of intestinal obstruction in infants.[10] An Indian study also showed intussusception to be the most common acquired cause of 10. ${ }^{1}$ Delay in diagnosis may result in significant morbidity in terms of loss of intestinal viability thus leading to bowel resection. Various studies have been carried out to find the risk factors for the loss of intestinal viability. Older age, longer duration of symptoms, palpable abdominal mass, and presence of pathological lead point were identified as a risk factor for bowel resection in childhood intestinal obstruction by a study carried out by C.WY.Wong et al. ${ }^{11}$ In a Pakistani study duration of symptoms more than 24 hours and anemia (hemoglobin $<10 \mathrm{~g} / \mathrm{dl}$ and haematocrit $<30 \%$ ) were found to be the risk factors for bowel resection. This study showed $36.3 \%$ population had to undergo bowel resection. ${ }^{9}$ In our study 10 out of 40 patients $(25 \%)$ had bowel resection and most of them had bowel gangrene due to delayed presentation.

Meckel's diverticulum the most common congenital anomaly of the intestinal tract and is notorious for causing intestinal obstruction in many ways. ${ }^{12}$ A band from diverticulum to anterior abdominal wall can be a direct source of mechanical obstruction. It can cause rotation leading to volvulus. Meckel's diverticulum can be a lead point for intussusception, thus causing intestinal obstruction. Perforation of inflamed Meckel's diverticulum can cause adhesive obstruction. Aziz DAA et al described a case of large, mobile and floppy Meckel's diverticulum causing intestinal obstruction by direct compression of the adjacent small bowel ${ }^{12}$. In our study, only $11(4.8 \%)$ had Meckel's diverticulum as a cause of intestinal obstruction. An Indian study showed $7.5 \%$ incidence of Meckel's diverticulum with band causing bowel obstruction.

Perforation of the gastrointestinal tract, solid organ injuries, and vascular injuries are dreadful complications of blunt abdominal trauma. In developing countries, children are more vulnerable to blunt abdominal trauma secondary to motor vehicle accidents, fall from height and bicycle accidents. The reported incidence of gastrointestinal perforation secondary to blunt abdominal trauma is $1 \%$ to $14 \%$. Early diagnosis and prompt intervention are necessary to avoid morbidity and mortality. ${ }^{13}$

Worm infestations is the leading cause of intestinal obstruction in developing countries and Ascariasis the common infestation in tropical areas, in children who belong to low socioeconomic status with poor hygiene. ${ }^{14}$ One and a half billion people are infected with ascaris worldwide constituting about $25 \%$ of world population. ${ }^{14}$ Globally there are about 730,000 cases of intestinal obstruction and 11,000 deaths annually worldwide. ${ }^{15}$ Astudy from Kashmir shows worm infestation to be the commonest cause of bowel obstruction (63.2\%). ${ }^{14}$ However, in our study, only $9(2.6 \%)$ patients had bowel obstruction secondary to worm infestation. Another study from Kenya showed ascariasis to be the leading cause of bowel obstruction accounting for $44.2 \%$ of the study cohort. ${ }^{10}$

In our study infection rate was high in neonatal age group during post-operative period i.e. Sepsis in $61(19.3 \%)$ patients, surgical site infection in 24 (7.6\%). A study from Bangladesh also showed sepsis to be the most common post operative complication in neonatal $10 .{ }^{3}$ Several factors may contribute to this high infection rate. All of the neonates were managed in the ward because of non-availability of surgical neonatal intensive care unit. Neonates are more prone to get cross infections and opportunistic infections. Under such circumstances, owing to limited physiological reserves than older children, thus show high infection rate in our study. Surgical site infection and wound dehiscence may be related to surgical technique by residents and may also be because most of the surgeries were performed during emergency hours when operation theatre/hospital facilities are at their lowest.

Post operative Sepsis in neonates was the major contributor of neonatal mortality in our study. Evaluation of factors contributing to sepsis and overcoming them, improvement in surgical techniques, availability of surgical neonatal intensive care unit, provision of adequate and well trained staff can be helpful in increasing the survival of pediatric $\mathrm{IO}$ patients especially neonates. 


\section{CONCLUSIONS}

Pediatric intestinal obstruction is the common surgical emergency encountered in hospitals and causes of intestinal obstruction vary according to the age and geographical area. Accurate and timely diagnosis of the cause of intestinal obstruction along with prevention and treatment of sepsis can reduce undue morbidity and mortality. Surgical Management of intestinal obstruction depends upon the cause and tailored according to the circumstances and condition of the patient.

\section{COMPETING INTERESTS}

The authors declare no competing interests

\section{REFERENCES}

1. Maheshwari M, Tanwani R, Patel M, Joshi A, Jain R, Praneeth $\mathrm{E}$. Intestinal obstruction in pediatric age group: A clinico-pathological study. Ann Int Med Dent Res. 2016;2(6):28-30.

2. Ooko PB, Wambua P, Oloo M, Odera A, Topazian HM, White R. The spectrum of paediatric intestinal obstruction in Kenya. Pan Afr Med J. 2016;24(1):439.

3. Saha AK, Ali MB, Biswas SK, Sharif HZ, Azim A. Neonatal intestinal obstruction: patterns, problems and outcome. Bangladesh Medical Journal Khulna. 2012;45(1-2):6-10.

4. Ali I, Mufti GN, Bhat NA, Baba AA, Sheikh KA, Hamid R, et al. Assessment of predictors of mortality in neonatal intestinal obstruction. Journal of Neonatal Surgery. 2018;7(1):2-8

5. Shah M, Gallaher J, Msiska N, McLean SE, Charles AG. Pediatric intestinal obstruction in Malawi: characteristics and outcomes. The American Journal of Surgery. 2016;211(4):722-6.

6. Mohammed M, Amezene T, Tamirat M. Intestinal Obstruction in Early Neonatal Period: A 3-Year Review Of Admitted Cases from a Tertiary Hospital in Ethiopia. Ethiop J Health Sci. 2017;27(4):393400.

7. Mitul AR. Congenital Neonatal Intestinal Obstruction. J Neonatal Surg. 2016;5(4):41-8.

8. Chalya PL, Kayange NM, Chandika AB. Childhood intussusceptions at a tertiary care hospital in northwestern Tanzania: a diagnostic and therapeutic challenge in resource-limited setting. Ital J Pediatr. 2014;40(1):28-33.

9. Nisar MU, Sikander S, Khan NA, Javed N, Chaudhary MA, Saif MAA. Determinants of Bowel Resection in Childhood Intussusception. Journal of Ayub Medical College Abbottabad. 2020;32(1):912.

10. Ooko PB, Wambua P, Oloo M, Odera A, Topazian HM, White R. The Spectrum of Paediatric Intestinal Obstruction in Kenya. Pan Afr Med J. 2016;24:4349.

11. Wong CW, Jin S, Chen J, Tam PK, Wong KK. Predictors for bowel resection and the presence of a pathological lead point for operated childhood intussusception: A multi-center study. J Pediatr Surg. 2016;51(12):1998-2000.
Aziz DA, Sehat SI, Osman M, Zaki FM. Neonatal intestinal obstruction secondary to a floppy Meckel's diverticulum successfully treated by minimal access surgery. BMJ Case Rep. 2012;2012.

13. Ciftci AO, Tanyel FC, Salman AB, Buyukpamukcu N, Hicsonmez A. Gastrointestinal tract perforation due to blunt abdominal trauma. Pediatr Surg Int. 1998;13(4):259-64.

14. Shiekh K, Baba A, Ahmad S, Shera A, Patnaik R, Sherwani A. Mechanical small bowel obstruction in children at a tertiary care centre in Kashmir. Afr J Paediatr Surg. 2010;7(2):81-5.

15. Mishra PK, Agrawal A, Joshi M, Sanghvi B, Shah H, Parelkar SV. Intestinal obstruction in children due to Ascariasis: a tertiary health centre experience. Afr J Paediatr Surg. 2008;5(2):65-70.
DATA SHARING STATEMENT: The data that support the findings of this study are available on request from the corresponding author. The data are not publicly available due to privacy or ethical restrictions.

CONFLICT OF INTEREST: Authors declare no conflict of interest.

GRANTED SUPPORT AND FINANCIAL DISCLOSURE:
Nil

\section{AUTHOR'S CONTRIBUTION}

Following authors have made substantial contributions to the manuscript as under

Chaudhry AR: Concept and design of study, Collection of data, statistical analysis

Nisar MU, Khan A:Writing of manuscript, critical review of manuscript

Akhtar N, Sikandar S:Analysis and interpretation of data, statistical analysis

Khan NA: Data collection, bibliography

Authors agree to be accountable for all aspects of the work in ensuring that questions related to the accuracy or integrity of any part of the work are appropriately investigated and resolved. 